\title{
A Predictive Control Model for Master Slave Robotic Manipulator with RBF Neural Network
}

\author{
Youjian Lei \\ Department of Electronic Information Technology, Jiangmen Polytechnic, Jiangmen 529090, \\ China
}

Received: December 29, 2020. Revised: June 30, 2021. Accepted: July 14, 2021. Published: July 19, 2021.

\begin{abstract}
In recent years, manipulator control has been widely concerned, and its uncertainty is one of the focuses. As we all know, the manipulator is a MIMO nonlinear system, which has the characteristics of severe variable coupling, large time-varying amplitude of parameters and high degree of nonlinearity. Therefore, a lot of uncertain factors must be considered when designing the control algorithm of manipulator system. The predictive control algorithm adopts online rolling optimization, and in the process of optimization, feedback correction is carried out by the difference between the actual output and the reference output. It can iterate the predictive model and suppress the influence of some uncertain disturbances to a certain extent. Therefore, the design of predictive controller for robot is not only of theoretical significance, but also of great practical significance. The trajectory tracking problem is proposed in this paper, and a predictive control method for master slave robotic manipulator with sliding mode controller is designed. In addition, when external disturbances occurred, the approximation errors are compensated by the proposed control method. Finally, The results demonstrate that the stability of the controllers can be improved for the trajectory tracking errors.
\end{abstract}

Keywords-Predictive Control, Master Slave Robotic Manipulator, Sliding Mode Controller, Approximation Errors

\section{INTRODUCTION}

$\mathrm{T}$ he master and the slave robot communicate through a network in bilateral teleoperation system [1-2]. On the one hand, the operator from a remote terminal manipulates a master robot to perform certain actions, and these action commands are transmitted to the slave robot through a communication channel to direct it to accomplish certain missions. On the other hand, using devices like tactile receptor, sensor, or webcam, a robot can give real-time feedback about the actual conditions on the site as well as the forces to the operator, making him feel personally on the scene. Thus, telerobot can replace human beings to perform disaster rescue mission, and minimally invasive surgery, etc., in some hazardous or unreachable circumstances. To the solve the problems existing in teleoperation system, scholars both at home and abroad have started to do researches in this area from a long time ago, and proposed various control algorithms.

The communication time delay in the signal transmission process is one of the main reasons leading to the instability of a system [3-5]. In 2020, Literature [6] made an analogy between teleoperation system and circuit network, and proposed a two-port network theory. He noted that the instability of a system was mainly due to the activeness of transmission line resulting from communication time delay, and the key to solve this problem was to make it acquire the properties of passive transmission line by controlling communication. Hereafter, based on the two-port network theory, many scholars tired to compensate for communication time delay by designing control algorithm. The most representative was the scattering-matrix theory proposed by Tapar J , Kishen S and Emani N K [7], who designed a compensator for independent and continuous time-delay to maintain the stability of a system. GK Mertin, M Oldenburger and E Richter put forward the notion of wave variable, and applied energy flow theory to solving the activeness of transmission line, maintaining the stability of a system with random time delay [8]. But these algorithms would result in the position tracking error, and with the increase of time delay, the operability of a system decreases.

In teleoperation system, the force between robot and environment as real-time feedback is reported to the operator, giving him the feeling that he himself is operating in the environment, and this is called the transparency of the system [9-11]. The control system based on position error has simple structure and does not need any force sensor. The multiplication of position error and a proportionality factor can be a direct force feedback to the operator. Direct force feedback control has better force tracking performance with the operators getting as a feedback, the force between a robot and the environment, which is accurately measured by a sensor. Literature [12] have successively proposed and developed the four-channel control architecture, in which the information as control signal was transmitted, and can achieve ideal transparency. However, the lack of position information and the minor mismatches of initial position will all results in 
the oscillation of a teleoperation system and damage its stability.

To solve the problem of the teleoperation system in space or deep ocean, the method of tele-programming and autonomous working of slave robot are adopted for supervision and control of the system. To avoid the uncertainty about the length of time delay in network communication. Literature [13] proposed the event-based control method, in which there was no need for controller design to directly depend on the length of time delay, instead the non-time reference variable was taken as input. Many other control methods, including robust control, fuzzy control, linear quadratic optimal control, virtual reality control, have also been widely applied to the teleoperation system to meet the requirements of stability and transparency [14-15].

\section{MATHEMATICAL MODEL OF THE CONTROLLED OBJECT}

Suppose $q_{m}, q_{s}$ respectively represent the position information of robot, $\tau_{m}, \tau_{s}$ are the output torques of controller, $F_{h}, F_{e}$ respectively represent the force of the operator and the environment. When the system operates, the operator exerts a force $F_{h}$ upon the master robot, and its position $q_{m}$ therefore changes. The applied force and the position information of the mater robot are all transmitted to the remote terminal through communication channel as input to the controller. While the controller outputs torque $\tau_{s}$, controls the salve robot's movement and changes its position $q_{s}$. Meanwhile, there will also be a force $F_{e}$ on the slave robot from the environment, as it gets in contact with external objects. The force feedback from the environment and the position information of slave robot are again transmitted back as input to the master controller. The controller outputs torque $\tau_{m}$, which is applied on the master robot to make the operator feel the force.

A teleoperation system with only the position information of master robot and force being transmitted to the slave robot is a unilateral system [16-18]. It is bilateral teleoperation system, if it can not only transmit the position information, but can also receive and feed back the position information. Bilateral system has better transparency [19], and the operator can truly experience the force, and seem to be personally on the scene by accurately feeling the rigidity, and mass, etc., of the object touched. Bilateral teleoperation system enables the operator to have a better perception, and therefore has more practical value [20].

The dynamic model of master/slave robot joint space with n-degree-of-freedom can be represented as

$$
\left\{\begin{array}{l}
M_{m}\left(q_{m}\right) \ddot{q}_{m}+C_{m}\left(q_{m}, \dot{q}_{m}\right) \dot{q}_{m}+F_{m} \dot{q}_{m}+f_{c m}\left(\dot{q}_{m}\right)+\tau_{d m}+G_{m}\left(q_{m}\right)=\tau_{m}+F_{h} \\
M_{s}\left(q_{s}\right) \ddot{q}_{s}+C_{s}\left(q_{s}, \dot{q}_{s}\right) \dot{q}_{s}+F_{s} \dot{q}_{s}+f_{c s}\left(\dot{q}_{s}\right)+\tau_{d s}+G_{s}\left(q_{s}\right)=\tau_{s}+F_{e}
\end{array}\right.
$$

where $M_{m}\left(q_{m}\right), M_{s}\left(q_{s}\right)$ mean the inertia matrix,
$C_{m}\left(q_{m}, \dot{q}_{m}\right), C_{s}\left(q_{s}, \dot{q}_{s}\right)$ mean the Coriolis matrix, $F_{m}, F_{s}$ is the viscous friction coefficient, $f_{c m}\left(\dot{q}_{m}\right), f_{c s}\left(\dot{q}_{s}\right)$ is Coulomb's friction coefficient, $\tau_{d m}, \tau_{d s}$ are unknown external disturbances, $G_{m}\left(q_{m}\right), G_{s}\left(q_{s}\right)$ are gravity torques, $F_{h}, F_{e}$ mean the input torques, $\tau_{m}, \tau_{s}$ mean the controller torques, $q_{m}, q_{s}$ mean the angular position matrix, $\dot{q}_{m}, \dot{q}_{s}$ mean the angular-velocity matrix, and $\ddot{q}_{m}, \ddot{q}_{s}$ mean the angular acceleration matrix.

In this study, the viscous coefficient and Coulomb's friction coefficient are selected according the specific conditions of the master and the slave, and Coulomb's friction coefficient $f_{c}(\dot{q})$ is a bounded piecewise continuous function.

The master/slave robot with 2-degree of freedom, two-link and rotary joints are studied in this paper, and the robot model is shown Figure 1.

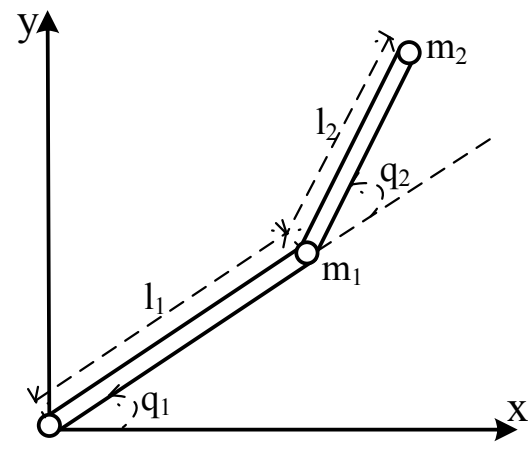

Fig. 1 The robot model with 2-degree of freedom, two-link and rotary joints

In Figure $1, l_{1}, l_{2}$ are the lengths of the two links, $m_{1}, m_{2}$ are the links' mass, $q_{1}, q_{2}$ are the joint angular positions of the two links.

For a robot as shown in the above figure, the parameters of the system model are as follows.

$$
\begin{aligned}
& \mathrm{M}_{\mathrm{m}}=M_{s}=\left\lfloor\begin{array}{ll}
M_{11} & M_{12} \\
M_{21} & M_{22}
\end{array}\right\rfloor, C_{m}=C_{s}=\left\lfloor\begin{array}{ll}
C_{11} & C_{12} \\
C_{21} & C_{22}
\end{array}\right\rfloor, \\
& G_{m}=G_{s}=\left\lfloor\begin{array}{l}
g_{1} \\
g_{2}
\end{array}\right\rfloor, l_{1}=l_{2}=l . \\
& M_{11}=\frac{1}{3} l^{2} m_{1}+\frac{4}{3} l^{2} m_{2}+l^{2} m_{2} \cos \left(q_{2}\right) \quad M_{21}=M_{12} \\
& M_{12}=\frac{1}{3} l^{2} m_{2}+\frac{1}{2} l^{2} m_{2} \cos \left(q_{2}\right) \quad M_{22}=\frac{1}{3} l^{2} m_{2} \\
& C_{11}=-\frac{1}{2} l^{2} \sin \left(q_{2}\right) \dot{q}_{2} \quad C_{21}=\frac{1}{2} l^{2} \sin \left(q_{2}\right) \dot{q}_{1} \\
& C_{12}=-\frac{1}{2} l^{2} \sin \left(q_{2}\right)\left(\dot{q}_{1}+\dot{q}_{2}\right) \quad C_{22}=0
\end{aligned}
$$




$$
\begin{aligned}
& g_{1}=g\left(\frac{1}{2} l m_{2} \cos \left(q_{1}+q_{2}\right)+\left(\frac{1}{2} m_{1}+m_{2}\right) l \cos \left(q_{1}\right)\right) \\
& g_{2}=\frac{1}{2} g l m_{2} \cos \left(q_{1}+q_{2}\right) \\
& F_{i} \dot{q}_{i}+f_{c i}\left(\dot{q}_{i}\right)=\left[\begin{array}{c}
f_{d 1} \dot{q}_{1}+k_{1} \operatorname{sgn}\left(\dot{q}_{1}\right) \\
f_{d 2} \dot{q}_{2}+k_{2} \operatorname{sgn}\left(\dot{q}_{2}\right)
\end{array}\right](i=m, s) \\
& \tau_{d i}=\left[\begin{array}{c}
0.3 q_{1} \dot{q}_{1} \sin t \\
0.3 q_{2} \dot{q}_{2} \sin t
\end{array}\right](i=m, s)
\end{aligned}
$$

\section{DESIGN OF CONTROLLER}

The design scheme in this study is that the various signals of the robot are transmitted in the communication network. The operator manipulates the master robot sending position command which are transmitted as its position reference to the slave robot after $T_{m}$ second delay in communication network. At the same time, the various signals of the slave robot will link up the master robot after a time delay of $T_{s}$ seconds to jointly control its motion.

The predictive control model is designed separately to track their trajectories. The transmission delay in communication channel will create difficulties for system control. In the part of neural network, Gaussian Neural Network approximation is adopted to maximally estimate the input/output system. The sliding mode control and adaptive control are adopted to compensate the approximation error and the external disturbance. In a certain period of time, trajectory tracking errors of master and slave robot asymptotically converge to 0 .

Firstly, define two sliding mode variables $r_{m}$ and $r_{s}$

$$
\begin{aligned}
& r_{m}=\dot{e}_{m}+\lambda_{m} e_{m} \\
& r_{s}=\dot{e}_{s}+\lambda_{s} e_{s}
\end{aligned}
$$

where $\lambda_{m}, \lambda_{s}$ is the positive diagonal matrix, and error signals $e_{m}$ and $e_{s}$ are defined as

$$
\begin{aligned}
& e_{m}=q_{s}\left(t-T_{s}\right)-q_{m} \\
& e_{s}=q_{m}\left(t-T_{m}\right)-q_{s}
\end{aligned}
$$

where, $T_{m}$ and $T_{s}$ are the time delay of signal input.

When $r_{m}$ and $r_{s}$ are synchronization variables, the dynamic model of formula (1) operating system can be rewrite,

$$
\left\{\begin{array}{l}
M_{m}\left(q_{m}\right) \dot{r}_{m}+C_{m}\left(q_{m}, \dot{q}_{m}\right) r_{m}=f_{m}\left(X_{m}\right)-\tau_{m}-F_{h}+\tau_{d m} \\
M_{s}\left(q_{s}\right) \dot{r}_{s}+C_{s}\left(q_{s}, \dot{q}_{s}\right) r_{s}=f_{s}\left(X_{s}\right)-\tau_{s}-F_{e}+\tau_{d s}
\end{array}\right.
$$

where

$$
\begin{aligned}
& X_{m}=\left[\ddot{q}_{s}^{T}\left(t-T_{s}\right), \dot{q}_{s}^{T}\left(t-T_{s}\right), q_{s}^{T}\left(t-T_{s}\right), \dot{q}_{m}^{T}, q_{m}^{T}\right]^{T} \\
& X_{s}=\left[\ddot{q}_{m}^{T}\left(t-T_{m}\right), \dot{q}_{m}^{T}\left(t-T_{m}\right), q_{m}^{T}\left(t-T_{m}\right), \dot{q}_{s}^{T}, q_{s}^{T}\right]^{T} .
\end{aligned}
$$

Due to its inner approximation ability, neutral network has been widely used to approximate unknown nonlinear system. Approximating a continuous function $f_{1}(X): R^{q} \rightarrow R^{2}$ is represented as

$$
f_{1}(X)=W^{T} \varphi(X)+\varepsilon(X)
$$

Where $X$ is input vector, $W$ is weight matrix, $\varphi(X)$ is the network function.

The neural network approximation function exists

$$
f_{1}(X)=W^{* T} \varphi(X)+\varepsilon_{1}^{*}(X),\left\|\varepsilon_{1}^{*}(X)\right\| \leq \varepsilon_{N 1}
$$

where $W^{*}$ is the ideal weight of approximation function with $W=W^{*}, \varepsilon_{1}^{*}(X)$ is approximation error, and $\varepsilon_{N 1}$ is the upper bound of approximation error.

According to the theorem of neural network approximation, $f_{m}\left(X_{m}\right)$ and $f_{s}\left(X_{s}\right)$ can be approximately represented by $\hat{f}_{m}\left(X_{m}\right)$ and $\hat{f}_{s}\left(X_{s}\right)$. Therefore,

$$
\begin{gathered}
\hat{f}_{m}\left(X_{m}\right)=\hat{W}_{m}^{T} \varphi_{m}\left(X_{m}\right) \\
\hat{f}_{s}\left(X_{s}\right)=\hat{W}_{s}^{T} \varphi_{s}\left(X_{s}\right)
\end{gathered}
$$

where $\hat{W}_{m}, \hat{W}_{s}$ is the weight parameter of RBF neural network, and $\varphi_{m}\left(X_{m}\right), \varphi_{s}\left(X_{s}\right)$ is RBF neural network activation function.

Therefore

$$
\begin{aligned}
& \bar{\varepsilon}_{m}^{*}\left(X_{m}\right)=f_{c m}\left(\dot{q}_{m}\right)+\varepsilon_{m}^{*}\left(X_{m}\right) \\
& \bar{\varepsilon}_{s}^{*}\left(X_{s}\right)=f_{c s}\left(\dot{q}_{s}\right)+\varepsilon_{s}^{*}\left(X_{s}\right)
\end{aligned}
$$

In previous researches, controller is usually designed with errors of external known disturbance. There are cases where errors of external disturbance are unknown, and therefore a method based on sliding mode and adaptive control technique is proposed in this study to compensate for these uncertainties.

The neutral network-based controller design is as follows.

$$
\begin{aligned}
& \tau_{m}=\hat{f}_{m}\left(X_{m}\right)+K_{m} r_{m}+\frac{r_{m}}{\left\|r_{m}\right\|+e^{-a_{m} t}} \theta_{m} \\
& \tau_{s}=\hat{f}_{s}\left(X_{s}\right)+K_{s} r_{s}+\frac{r_{s}}{\left\|r_{s}\right\|+e^{-a_{s} t}} \theta_{s}
\end{aligned}
$$

The first section of the controller is the neural network control part by the law of neural network control,

$$
\begin{aligned}
& \dot{\hat{W}}_{m}=\Lambda_{m} \varphi_{m}\left(X_{m}\right) r_{m}^{T} \\
& \dot{\hat{W}}_{s}=\Lambda_{s} \varphi_{s}\left(X_{s}\right) r_{s}^{T}
\end{aligned}
$$

where $\Lambda_{m}, \Lambda_{s}$ a positive definite matrix.

The second section is the sliding-mode control part, as shown by equation (2). 
The third section is the adaptive control part. Where $\theta_{m}$ and $\theta_{s}$ can estimate the bounds of approximation error and external disturbances: $\theta_{o m}$ and $\theta_{o s}$,

$$
\left\|\bar{\varepsilon}_{m}^{*}\left(X_{m}\right)+\tau_{d m}\right\| \leq \theta_{o m},\left\|\bar{\varepsilon}_{m}^{*}\left(X_{s}\right)+\tau_{d s}\right\| \leq \theta_{o s}
$$

$K_{m}, K_{s}$ are positive numbers. According to the adaptive control law,

$$
\begin{aligned}
& \dot{\theta}_{m}=\frac{\left\|r_{m}\right\|^{2}}{\left\|r_{m}\right\|+e^{-a_{m} t}} \\
& \dot{\theta}_{s}=\frac{\left\|r_{s}\right\|^{2}}{\left\|r_{s}\right\|+e^{-a_{s} t}}
\end{aligned}
$$

where $a_{m}, a_{s}$ are positive numbers.

Applying the above controller in the free space (i.e. $F_{h}=F_{e}=0$ ), the tracking errors of master/slave robot's position and velocity asymptotically converge to zero with the time delay of $T_{m}$ and $T_{\mathrm{s}}$. More details can be seen in Section IV.

\section{THE STABILITY}

With Lyapunov function, the stability and trajectory tracking performance of the model are analyzed. Firstly, construct a Lyapunov function

$$
\begin{aligned}
V= & \frac{1}{2} r_{m}^{T} M_{m}\left(q_{m}\right) r_{m}+\frac{1}{2} \operatorname{trace}\left(\tilde{W}_{m}^{T} \Lambda_{m}^{-1} \tilde{W}_{m}\right)+\frac{1}{2}\left(\theta_{m}-\theta_{o m}\right)^{2} \\
& +\frac{1}{2} r_{s}^{T} M_{s}\left(q_{s}\right) r_{s}+\frac{1}{2} \operatorname{trace}\left(\tilde{W}_{s}^{T} \Lambda_{m}^{-1} \tilde{W}_{s}\right)+\frac{1}{2}\left(\theta_{m}-\theta_{o m}\right)^{2}
\end{aligned}
$$

Perform differential calculation of the above function, equation (10) which is the neural network control law into it,

$$
\begin{aligned}
\dot{V}= & r_{m}^{T}\left(f_{m}\left(X_{m}\right)-\hat{f}_{m}\left(X_{m}\right)-K_{m} r_{m}-\frac{r_{m}}{\left\|r_{m}\right\|+e^{-a_{m} t}} \theta_{m}+\tau_{d m}\right) \\
& +\left(\theta_{m}-\theta_{o m}\right) \dot{\theta}_{m}-\operatorname{trace}\left(\tilde{W}_{m}^{T} \varphi_{m}\left(X_{m}\right) r_{m}^{T}\right) \\
& +r_{s}^{T}\left(f_{s}\left(X_{s}\right)-\hat{f}_{s}\left(X_{s}\right)-K_{s} r_{s}-\frac{r_{s}}{\left\|r_{s}\right\|+e^{-a_{s} t}} \theta_{s}+\tau_{d s}\right) \\
& +\left(\theta_{s}-\theta_{o s}\right) \dot{\theta}_{s}-\operatorname{trace}\left(\tilde{W}_{s}^{T} \varphi_{s}\left(X_{s}\right) r_{s}^{T}\right)
\end{aligned}
$$

Substituting equation (12) which is the adaptive control law into it,

$$
\begin{aligned}
\dot{V}= & r_{m}^{T}\left(\bar{\varepsilon}_{m}^{*}\left(X_{m}\right)+\tau_{d m}\right)-\frac{\left\|r_{m}\right\|^{2}}{\left\|r_{m}\right\|+e^{-a_{m} t}} \theta_{m}+\left(\theta_{m}-\theta_{o m}\right) \frac{\left\|r_{m}\right\|^{2}}{\left\|r_{m}\right\|+e^{-a_{m} t}} \\
& +r_{s}^{T}\left(\bar{\varepsilon}_{s}^{*}\left(X_{s}\right)+\tau_{d s}\right)-\frac{\left\|r_{s}\right\|^{2}}{\left\|r_{s}\right\|+e^{-a_{s} t}} \theta_{s}+\left(\theta_{s}-\theta_{o s}\right) \frac{\left\|r_{s}\right\|^{2}}{\left\|r_{s}\right\|+e^{-a_{s} t}} \\
& -r_{m}^{T} K_{m} r_{m}-r_{s}^{T} K_{s} r_{s}
\end{aligned}
$$

Substituting

$\left\|\bar{\varepsilon}_{m}^{*}\left(X_{m}\right)+\tau_{d m}\right\| \leq \theta_{o m},\left\|\bar{\varepsilon}_{s}^{*}\left(X_{s}\right)+\tau_{d s}\right\| \leq \theta_{o s} \quad$ into it,

$$
\begin{aligned}
\dot{V} \leq & \left\|r_{m}\right\| \theta_{o m}-\frac{\left\|r_{m}\right\|^{2}}{\left\|r_{m}\right\|+e^{-a_{m} t}} \theta_{o m}+\left\|r_{s}\right\| \theta_{o s}-\frac{\left\|r_{s}\right\|^{2}}{\left\|r_{s}\right\|+e^{-a_{s} t}} \theta_{o s} \\
& -r_{m}^{T} K_{m} r_{m}-r_{s}^{T} K_{s} r_{s}
\end{aligned}
$$

$\dot{V} \leq-r_{m}^{T} K_{m} r_{m}+e^{-a_{m} t} \theta_{\text {om }}-r_{s}^{T} K_{s} r_{s}+e^{-a_{s} t} \theta_{\text {os }}$

Calculating the integral from 0 to $t$,

$$
V(t)-V(0) \leq-\int_{0}^{t}\left(r_{m}^{T} K_{m} r_{m}+r_{s}^{T} K_{s} r_{s}\right) d t+\theta_{o m} \int_{0}^{t} e^{-a_{m} t} d t+\theta_{o s} \int_{0}^{t} e^{-a_{s} t} d t
$$

Since $V(T) \geq 0$ and $\int_{0}^{\infty} e^{-a_{m} t} d t<\infty, \int_{0}^{\infty} e^{-a_{s} t} d t<\infty$,

$$
\begin{aligned}
& \limsup _{t \rightarrow \infty} \frac{1}{t} \int_{0}^{t}\left(K_{m}\left\|r_{m}\right\|^{2}+K_{s}\left\|r_{s}\right\|^{2}\right) d t \\
& \quad \leq\left(V(0)+\theta_{o m} \int_{0}^{t} e^{-a_{m} t} d t+\theta_{o s} \int_{0}^{t} e^{-a_{s} t} d t\right) \lim _{t \rightarrow \infty} \frac{1}{t}=0
\end{aligned}
$$

When $t \rightarrow \infty, r_{m} \rightarrow 0, r_{s} \rightarrow 0, e_{m} \rightarrow 0, e_{s} \rightarrow 0$. The trajectory tracking errors of master and slave robot converge to zero with time delay $T_{m}$ and $T_{s}$. The stability and trajectory tracking performance of this controller is proved.

\section{SIMULATION}

In simulation, suppose the external input $F_{h}=F_{e}=0$, and the parameters selected are as follows

$$
\begin{gathered}
m_{1}=m_{2}=1 \mathrm{~kg}, l=1 \mathrm{~m}, g=9.81 \mathrm{~m} / \mathrm{s}^{2}, \\
f_{d 1}=3, f_{d 2}=4, k_{1}=5, k_{2}=4
\end{gathered}
$$

The design of parameter setting for this controller is

$$
\begin{gathered}
K_{m}=K_{s}=40, \Lambda_{m}=\Lambda_{s}=1, a_{m}=a_{s}=2, \\
\lambda_{m}=\lambda_{s}=5, T_{m}=T_{s}=0.6
\end{gathered}
$$

The initial values are

$$
\begin{array}{ll}
q_{m}(0)=\left[\begin{array}{ll}
0.4 \pi & 0.1 \pi
\end{array}\right]^{T}, & \dot{q}_{m}(0)=\left[\begin{array}{ll}
0 & 0
\end{array}\right]^{T} \\
q_{s}(0)=\left[\begin{array}{ll}
0.6 \pi & 0.3 \pi
\end{array}\right]^{T}, & \dot{q}_{s}(0)=\left[\begin{array}{ll}
0 & 0
\end{array}\right]^{T}
\end{array}
$$


Choose five neurons, and whose center and width are

$$
C=\left[\begin{array}{lllll}
2 & 3 & 2 & 3.3 & 3.5
\end{array}\right]^{T}, b=\left[\begin{array}{lllll}
4 & 7 & 8 & 5 & 4
\end{array}\right]^{T} .
$$

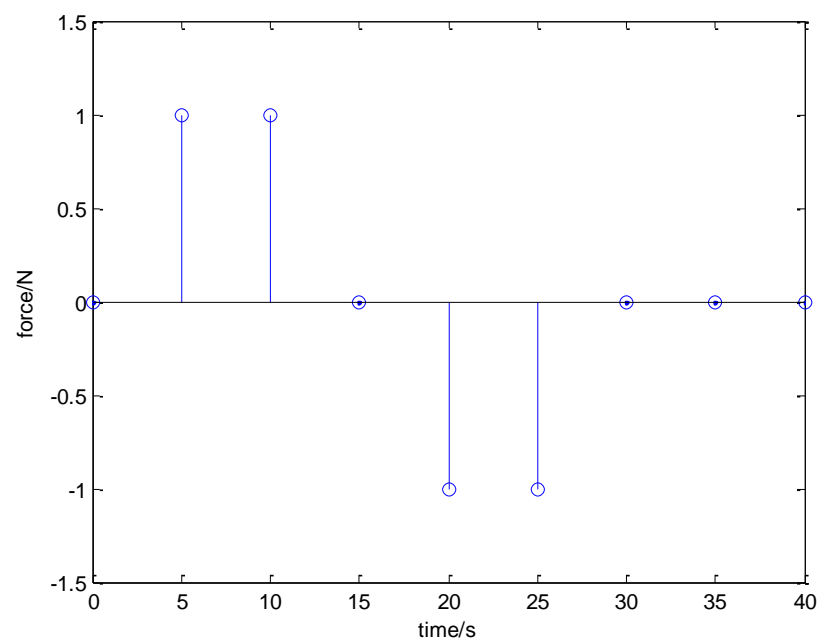

Figure 2. $F_{h}$ simulation curve

The input value of $F_{h}$ is shown in Figure 2. At 5th and 10th second, it is the pulse with amplitude 1, and at 20th and 25 th, it is the pulse with amplitude -1 .

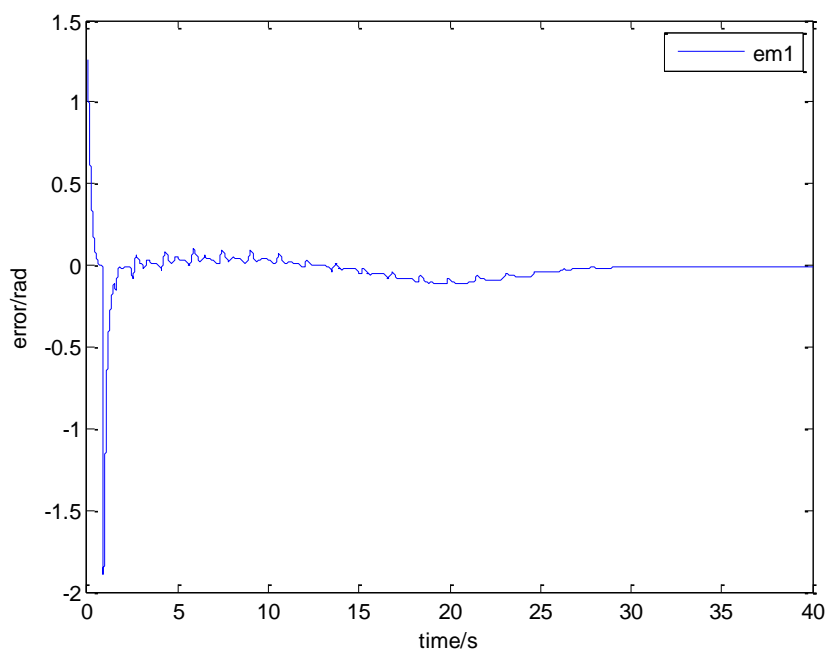

Figure 3. $e_{m}$ curve of master manipulator joints 1

Figure 3 to Figure 6 are error curves for the master/slave robot. Within the first 1 second after the beginning of simulation, due to time delay and neural network learning, great errors exist in the system, and then decrease quickly; after a short-time oscillating regulation, the position errors converge to zero, and the system reaches a stable state. This model has a stronger robustness than a single technology based controller.

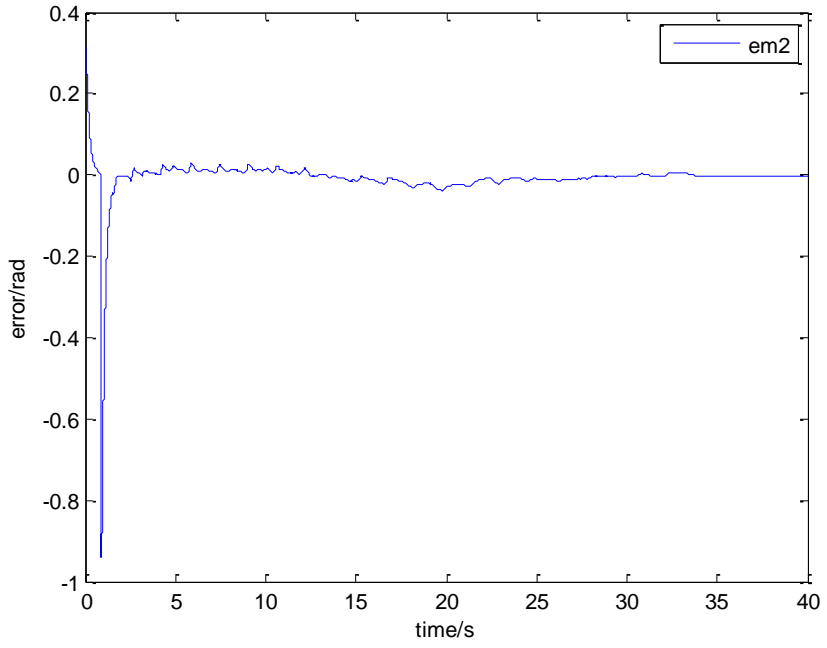

Figure 4. $e_{m}$ curve of master manipulator joints 2

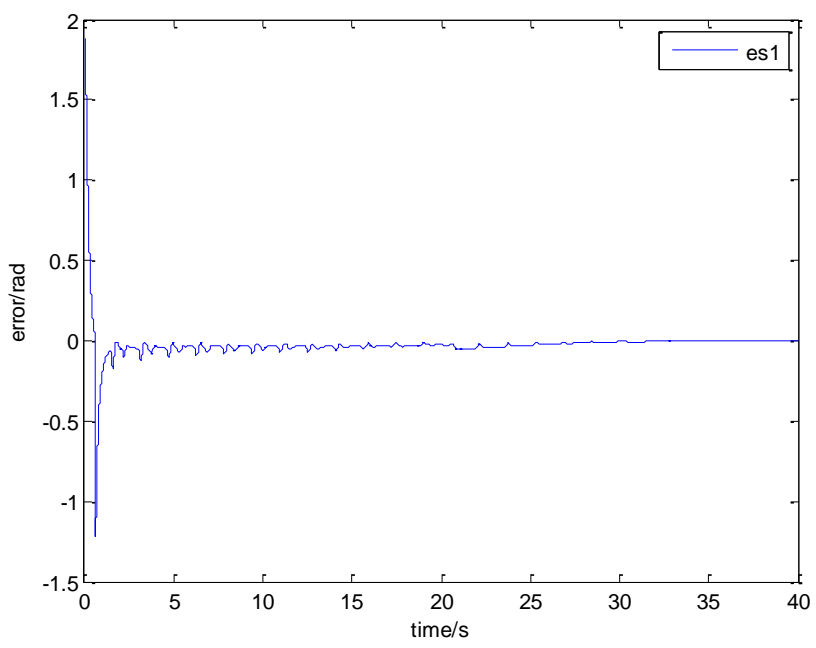

Figure 5. $e_{s}$ curve of salve manipulator joints 1

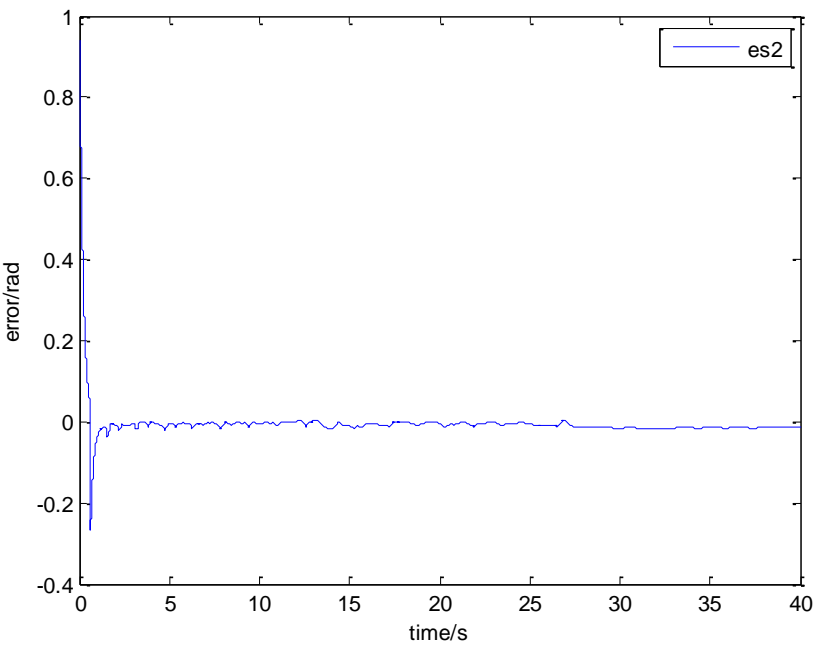

Figure 6. $e_{s}$ curve of salve manipulator joints 2 . 


\section{CONCLUSION}

A predictive control model was proposed for the master and the slave manipulator system of teleoperation. And then the theoretical derivation and system's stability proof were also given. The relationship between input and output of the complex nonlinear robot system was demonstrated by using function approximation. The error from the environment were compensated by sliding mode control adaptive control.

The future work can be based on looking for a more superior control strategy for the tracking control. A classical control method of PID is mainly used for master robotic manipulator, and a control method of machine learning is mainly used for slave robotic manipulator. And the Lyapunov function is designed to validate stability of the future model.

\section{REFERENCES}

[1] Wajiansyah A, Supriadi S. Implementasi Master-slave pada Embedded system menggunakan komunikasi RS485. ELKHA, 2020, 12, pp. 26-37.

[2] Barnett E, Gosselin C. Large-Scale 3D Printing With A CableSuspended Robot. Additive Manufacturing, 2015, 7, 27-44.

[3] Bento M . Design of a wide-area damping controller to tolerate permanent communication failure and time delay uncertainties. Energy Systems, 2021, 3, pp. 65-77.

[4] Wu T, Li Q, Bao X B , et al. Time-delay signature concealment in chaotic secure communication system combining optical intensity with phase feedback. Optics Communications, 2020, 475:126042.

[5] Wen S , Guo G . Sampled-Data Control for Connected Vehicles With Markovian Switching Topologies and Communication Delay. IEEE Transactions on Intelligent Transportation Systems, 2020, 21, pp. 29302942

[6] Chung H, Ma Q, Sayginer M, et al. A Packaged 0.01-26-GHz SingleChip SiGe Reflectometer for Two-Port Vector Network Analyzers. IEEE Transactions on Microwave Theory and Techniques, 2020, PP(99):1-15.

[7] Tapar J, Kishen S, Emani N K . Spectral singularities and asymmetric light scattering in PT-symmetric 2D nanoantenna arrays. Optics Letters, 2020, 45, pp. 375-382.

[8] GK Mertin, M Oldenburger, E Richter, Revised theory of entropy and reversible energy flow in galvanic cells. Journal of Power Sources, 2021, 482:228813.

[9] Van Den Berg J, Abbeel P, Goldberg K. LQG-MP: Optimized path planning for robots with motion uncertainty and imperfect state information. The International Journal of Robotics Research, 2019, 30(7): 895-913.

[10] Badmos T A, Omolaye P O, Mebawondu J , et al. Robot Path Planning Performance Evaluation of a Dynamic Environment. IOSR Journal of Electronics and Communication Engineering, 2020, 13, pp.19-26..

[11] Atia M G B, El-Hussieny H, Salah O. A Supervisory-Based Collaborative Obstacle-Guided Path Refinement Algorithm for Path Planning in Wide Terrains. IEEE Access, 2020,170: 257-266.

[12] D Sun, Naghdy F , Du H . Transparent four-channel bilateral control architecture using modified wave variable controllers under time delays. Robotica, 2016, 34, pp. 859-875.

[13] Cao Y , Wang S , Guo Z , et al. Stabilization of memristive neural networks with mixed time-varying delays via continuous/periodic eventbased control. Journal of the Franklin Institute, 2020, 357, pp.935-947.

[14] Aboutalebian B , Talebi H A, Etedali S, et al. Adaptive control of teleoperation system based on nonlinear disturbance observer ScienceDirect. European Journal of Control, 2020, 53, pp. 109-116.

[15] Ishiguro Y, Makabe T , Nagamatsu Y, et al. Bilateral humanoid teleoperation system using whole-body exoskeleton cockpit TABLIS. IEEE Robotics and Automation Letters, 2020, 99, 122-138.

[16] Islam M R , Protik P, Das S , et al. Mobile robot path planning with obstacle avoidance using chemical reaction optimization. Soft Computing, 2021,10, pp.1-28.
[17] Jung J W , Park J S , Kang T W , et al. Mobile Robot Path Planning Using a Laser Range Finder for Environments with Transparent Obstacles. Applied Sciences, 2020, 10, pp.2799.

[18] Martyshkin A I . Motion Planning Algorithm for a Mobile Robot with a Smart Machine Vision System. Nexo Revista Científica, 2021, 33, pp.651-671.

[19] Fethi, Matoui, Boumedyen, et al. Contribution to the path planning of a multi-robot system: centralized architecture. Intelligent Service Robotics, 2020, 13(1), pp. 147-158.

[20] Shih B Y, Chang H, Chen C Y. RETRACTED: Path planning for autonomous robots-a comprehensive analysis by a greedy algorithm. Journal of Vibration and Control, 2019, 19(1): 130-142.

\section{Creative Commons Attribution License 4.0 (Attribution 4.0 International, CC BY 4.0)}

This article is published under the terms of the Creative Commons Attribution License 4.0

https://creativecommons.org/licenses/by/4.0/deed.en_US 